\title{
Lifetimes of electrons in the Shockley surface state band of $\operatorname{Ag}(111)$
}

\author{
M. Becker,, * S. Crampin, ${ }^{2}$ and R. Berndt ${ }^{1}$ \\ ${ }^{1}$ Institut für Experimentelle und Angewandte Physik, \\ Christian-Albrechts-Universität zu Kiel, D-24098 Kiel, Germany \\ ${ }^{2}$ Department of Physics, University of Bath, Bath BA2 7AY, United Kingdom
}

(Dated: 31.08.2006)

\begin{abstract}
We present a theoretical many-body analysis of the electron-electron $(e-e)$ inelastic damping rate $\Gamma$ of electron-like excitations in the Shockley surface state band of $\mathrm{Ag}(111)$. It takes into account $a b$-initio band structures for both bulk and surface states. $\Gamma$ is found to increase more rapidly as a function of surface state energy $E$ than previously reported, thus leading to an improved agreement with experimental data.

PACS numbers:
\end{abstract}

\section{INTRODUCTION}

The quantitative understanding of the dynamics of electronic excitations in the Shockley state band at the (111) face of $\mathrm{Ag}$ has been the subject of a considerable number of research reports (for a review see Ref. [1]). Recent experimental studies used photoelectron spectroscopy and the scanning tunnelling microscope (STM) 2, 3, 4, 5, 6, 7, 8, 9].

Theoretically, many-body lifetime calculations using the GW approximation [5, 10] have clarified the role of electron-electron $(e-e)$ interactions as well as the relative importance of intraband and interband scattering and screening. The damping rate due to electron-phonon scattering is also quantitatively understood [11]. However, comparing theoretical with STM-derived experimental damping rates of electronic excitations with energies above the Fermi level $E_{F}$ show that the experimental decay rates increase more rapidly with increasing energy $E$. Recently a similar discrepancy between image state lifetimes measured by means of the STM and two-photon-photoemission spectroscopy was lifted 12 . It was shown that the applied electric field during the STM-measurement causes an increase in both the efficiency of the image state decay channels as well as their number. In the light of this work, Becker et al. theoretically investigated the impact of the STM-induced electric field on the inelastic $e-e$ damping rate of the electronand hole-like excitations in the Shockley surface state band at $\operatorname{Ag}(111)$ [13]. Their results indicate that under typical tunnelling conditions the STM does not significantly alter the surface state wave function and so previous STM-derived Shockley state lifetimes need not be corrected. Nevertheless, while experimental and calculated lifetimes agree quite well over a range of energies, a quantitative difference remains for unoccupied states of energies $E \gtrsim E_{F}+300 \mathrm{meV}$. The origin of this different energy dependence, which is still an open question, is addressed in this report.

*Electronic address: becker@physik.uni-kiel.de

\section{CALCULATION DETAILS}

The calculations presented here are based upon the approach developed by Chulkov et al. [14], and used widely in calculations of surface state dynamics 1]. Within this approach the damping rate or inverse lifetime of an excitation in the state $\psi(\mathbf{r})$ with energy $E$ is obtained from the expectation value of the imaginary part of the electron self-energy, $\Sigma\left(\mathbf{r}, \mathbf{r}^{\prime} ; E\right)$ :

$$
\Gamma=\tau^{-1}=-2 \int d \mathbf{r} \int d \mathbf{r}^{\prime} \psi^{*}(\mathbf{r}) \operatorname{Im} \Sigma\left(\mathbf{r}, \mathbf{r}^{\prime} ; E\right) \psi\left(\mathbf{r}^{\prime}\right) .
$$

Unless stated explicitly, atomic units are used throughout the text, i.e., $e^{2}=\hbar=m_{e}=1$.

In the GW approximation [15] of many-body theory the imaginary part of the self energy is calculated to first order in terms of the screened Coulomb interaction $W$ and the Green function $G$

$$
\operatorname{Im} \Sigma\left(\mathbf{r}, \mathbf{r}^{\prime} ; \epsilon\right)=-\frac{1}{\pi} \int_{E_{F}}^{\epsilon} d \epsilon^{\prime} \operatorname{Im} G\left(\mathbf{r}, \mathbf{r}^{\prime} ; \epsilon^{\prime}\right) \operatorname{Im} W\left(\mathbf{r}, \mathbf{r}^{\prime} ; \epsilon-\epsilon^{\prime}\right)
$$

Here we apply the widely used zeroth order approximation to the Green function in the spectral representation

$$
G\left(\mathbf{r}, \mathbf{r}^{\prime} ; \epsilon\right)=\sum_{i} \frac{\psi_{i}(\mathbf{r}) \psi_{i}^{*}\left(\mathbf{r}^{\prime}\right)}{\epsilon-E_{i}+\mathrm{i} \delta}
$$

where the $\psi_{i}(\mathbf{r})$ are one-electron eigenfunctions with energies $E_{i}$ and $\delta$ a positive infinitesimal. The screened Coulomb interaction is evaluated in the random phase approximation (RPA)

$$
\begin{aligned}
W\left(\mathbf{r}, \mathbf{r}^{\prime} ; \omega\right)= & v\left(\mathbf{r}-\mathbf{r}^{\prime}\right)+\int d \mathbf{r}_{1} \int d \mathbf{r}_{2} v\left(\mathbf{r}-\mathbf{r}_{1}\right) \\
& \times \chi^{0}\left(\mathbf{r}_{1}, \mathbf{r}_{2} ; \omega\right) W\left(\mathbf{r}_{2}, \mathbf{r}^{\prime} ; \omega\right)
\end{aligned}
$$

where $v$ is the bare Coulomb interaction and $\chi^{0}$ is the density-density response function of the non-interacting electron system. An explicit expression for $\chi^{0}$ can be found in Ref. [16]. 


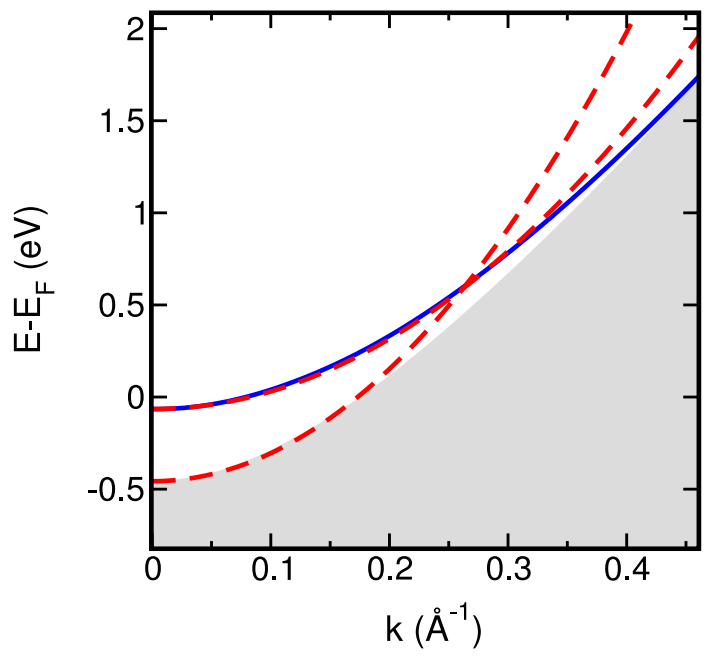

FIG. 1: (Color online) $\mathrm{Ag}(111)$ surface band structure. Shaded area and solid line are the projected bulk and surface state dispersion from ab-initio calculations which we use for calculating the $e$-e decay rate. The dashed lines are parabolic dispersions with effective masses $m^{*}=0.4$ (surface state band) and 0.25 (band edge).

The single particle states have been obtained by solving the Schrödinger equation using a one-dimensional pseudopotential varying in the direction perpendicular to the surface [17. The pseudopotential does not describe the $d$-electrons of the substrate, which are anyways too low in energy to play a significant role as final states for the decay, but their contribution to the screening is included via a polarisable background $[10,18]$. The electron-phonon contribution to the linewidth is taken to be constant $\Gamma_{\mathrm{e}-\mathrm{ph}}=3.7 \mathrm{meV}$ for energies in excess of $20 \mathrm{meV}$ above the Fermi level [1].

Previous calculations of the decay rate of the $\mathrm{Ag}(111)$ Shockley state have assumed parabolic dispersion with effective masses of $m^{*}=0.4$ and $m^{*}=0.25$ for the intrinsic surface state and the lower band edge respectively [8]. However, over the extended energy range of interest here, this approximation needs to be improved as evident from Fig. 1] Figure 1 shows the parabolic dispersions (dashed lines) for the surface state band and the band edge with effective masses as mentioned above. The grey shaded area and the solid line are the projected bulk and surface state dispersion, respectively, that are found from $a b$-initio calculations. The parabolic dispersion underestimates the gap between surface state band and band edge. We have therefore used the ab-initio dispersions for our lifetime calculations. As previously noted [8, 19] there are important changes in the shape of the surface state wave function with $k$, the surface wave vector, and we take these into account by recalculating the wave function for different $k$ with the pseudopotential parameters changed to take into account the appropriate $a b$-initio band edges and surface state energy.

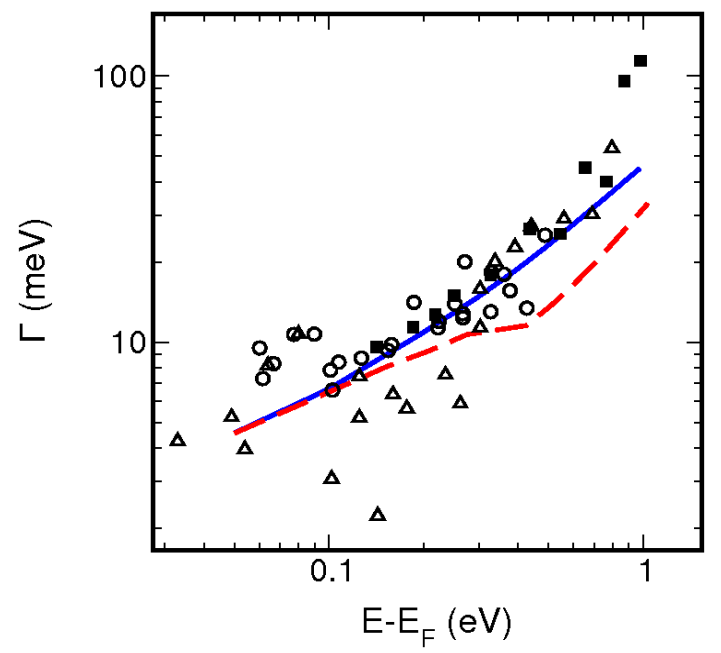

FIG. 2: (Color online) Comparison of the total Shockley surface state decay rates $\Gamma+\Gamma_{\mathrm{e}-\mathrm{ph}}$ for electrons at $\mathrm{Ag}(111)$ calculated using ab-initio dispersion relations (solid line) and previous published decay rates using parabolic dispersion (dashed line) from Ref. [1]. The black squares represent experimental data from Ref. [8], black triangles from Ref. [7] and black circles from Ref. [9]. The experimental data from Refs. [7, 8] have been multiplied by a factor 2 to compensate for an erroneous analysis in that work [20].

\section{RESULTS AND DISCUSSION}

We focus on the energy range between 0 and $1 \mathrm{eV}$ above the Fermi level where the surface state is energetically separated from the bulk states and thus is well defined. This energy range is of interest as according to Ref. [8] there is a cross-over in the primary decay channel from intraband to interband dominated decay. Our calculated results for the total damping rate $\Gamma+\Gamma_{\mathrm{e}-\mathrm{ph}}$ are shown in Fig. [2] as a solid line. For energies below $E-E_{F} \approx 0.1 \mathrm{eV}$ we find that our results for the inelastic $e$-e damping rate of excitations in the Shockley state band agree well with the inverse lifetime estimation from Echenique et al. (dashed line) 1] using similar methods but with an effective mass approximation. This agreement is due to the fact that the effective mass approximation gives a very good description of the projected ab-inito surface band structure for energies with $E-E_{F} \lesssim 0.1 \mathrm{eV}$ as evident from Fig. 11

For larger energies we find that the $e$-e damping rate as a function of energy increases more rapidly with the ab-initio dispersion than calculated with parabolic dispersions. This result can be understood as follows: For energies $E-E_{F} \gtrsim 0.1 \mathrm{eV}$ the parabolic dispersions underestimate the energy gap between surface state band and the bulk band edge. Consequently, the ab-initio dispersions reveal a reduced penetration of the surface state wave function into the interior of the crystal and an increased probability amplitude in the proximity of the metal surface. This modification leads to two competing effects. Since screening is reduced at the surface [5] 
intraband decay becomes more efficient. On the other hand, interband decay becomes less probable owing to the reduced overlap with bulk states. It turns out that the increase of intraband decay outweighs the decrease in interband transitions. The net result is a more steep increase of $\Gamma$ than obtained from parabolic dispersions. We thus find the intraband contribution to dominate up to $E-E_{F}=1 \mathrm{eV}$ where the interband and intraband contributions become similar in magnitude. Comparing to published experimental data (symbols in Fig. 21) the agreement is improved.

In summary, we have presented a theoretical manybody analysis of the inelastic $e-e$ lifetimes of electronic excitations in the Shockley surface state band of $\mathrm{Ag}(111)$. The calculations are based on the GW approximation us- ing a one-dimensional pseudopotential together with an $a b$-initio description of the projected surface band structure. We have shown that the consistency of experimental and theoretical derived damping rates is significantly improved when using ab-initio band structures instead of an effective mass approximation for calculating the decay rate.

\section{ACKNOWLEDGEMENTS}

M.B. and R.B. thank the Deutsche Forschungsgemeinschaft for financial support through SPP 1093.
[1] P. M. Echenique, R. Berndt, E. V. Chulkov, Th. Fauster, A. Goldmann, and U. Höfer, Surf. Sci. Rep. 52, 219 (2004).

[2] F. Reinert, G. Nicolay, S. Schmidt, D. Ehm, and S. Hüfner, Phys. Rev. B 63, 115415 (2001).

[3] J. Li, W.-D. Schneider, R. Berndt, O. R. Bryant, and S. Crampin, Phys. Rev. Lett. 81, 4464 (1998).

[4] L. Bürgi, O. Jeandupeux, H. Brune, and K. Kern, Phys. Rev. Lett. 82, 4516 (1999).

[5] J. Kliewer, R. Berndt, E. V. Chulkov, V. M. Silkin, P. M. Echenique, and S. Crampin, Science 288, 1399 (2000).

[6] J. Kliewer, R. Berndt, and S. Crampin, New J. Phys. 3, 22 (2001).

[7] K.-F. Braun and K.-H. Rieder, Phys. Rev. Lett. 88, 096801 (2002).

[8] L. Vitali, P. Wahl, M.A. Schneider, K. Kern, V.M. Silkin, E.V. Chulkov, and P.M. Echenique, Surf. Sci. 523, L47 (2003).

[9] H. Jensen, J. Kröger, R. Berndt, and S. Crampin, Phys. Rev. B 71, 155417 (2005).
[10] A. García-Lekue, J.M. Pitarke, E.V. Chulkov, A. Liebsch, and P.M. Echenique, Phys. Rev. B 68, 045103 (2003).

[11] A. Eiguren, B. Hellsing, E.V. Chulkov, and P.M. Echenique, Phys. Rev. B 67, 235423 (2003).

[12] S. Crampin, Phys. Rev. Lett. 95, 046801 (2005).

[13] M. Becker, S. Crampin, and R. Berndt, Phys. Rev. B 73, 81402 (2006).

[14] E.V. Chulkov, I. Sarria, V.M. Silkin, J.M. Pitarke, and P.M. Echenique, Phys. Rev. Lett. 80, 4947 (1998).

[15] L. Hedin, S. Lundqvist, Solid State Phys. 23, 1 (1969).

[16] Adolfo G. Eguiluz, Phys. Rev. B 31, 3303 (1985).

[17] E.V. Chulkov, V.M. Silkin, and P.M. Echenique, Surf. Sci. 437, 330 (1999).

[18] A. Liebsch, Phys. Rev. Lett. 71, 145 (1993).

[19] M.G. Vergniory, J.M. Pitarke, and S. Crampin, Phys. Rev. B 72, 193401 (2005).

[20] S. Crampin, J. Kröger, H. Jensen, and R. Berndt, Phys. Rev. Lett. 95, 029701 (2005). 